\title{
ON ODD-PRIMARY COMPONENTS OF LIE GROUPS
}

\author{
K. KNAPP
}

\begin{abstract}
The transfer map $t: \pi^{s}\left(P_{\infty} C\right) \rightarrow \pi^{s}\left(S^{9}\right)$ is represented by an element $\tau \in \pi_{s}^{-1}\left(P_{\infty} C^{+}\right)$. We compute the Adams-e-invariant of $\tau$ and use this and the splitting of the $p$-localization of $S^{1} \wedge P_{\infty} C$ into a wedge of $(p-1)$ spaces to prove that for a prime $p>5$ the $p$-component of the element $[G, \mathcal{E}]$ defined by a compact Lie group $G$ in $\pi_{*}^{s}$ is zero in the known part of stable homotopy.
\end{abstract}

1. The $e$-invariant of the transfer map. The easiest definition of the transfer $t_{S^{1}}$ : $\pi_{n}^{s}\left(P_{\infty} \mathrm{C}^{+}\right) \rightarrow \pi_{n+1}^{s}\left(S^{0}\right)$ is in terms of framed bordism: An element in $\pi_{n}^{s}\left(P_{\infty} \mathrm{C}^{+}\right)$is given by a framed manifold $(M, \Phi)$ together with an $S^{1}$-principal bundle $\xi$. The total space of $\xi$ together with the canonical framing constructed from $\Phi$ represents $t_{S^{1}}(M, \Phi)$. On finite skeletons we can represent $t_{S^{1}}$ by stable maps, which fit together to give an element $\tau$ in $\pi_{s}^{-1}\left(P_{\infty} \mathbf{C}^{+}\right)$. The map $\tau$ induces the transfer $t_{S^{1}}$ in stable cohomotopy and we have $\tau=t_{S^{1}}(1)$. Because we are always working in a fixed dimension in homology, we can avoid limit discussions by restricting to a finite skeleton. Let $\beta$ be the Bockstein map $\beta: \pi_{s}^{-2}\left(P_{\infty} \mathbf{C}^{+} ; \mathbf{Q} / \mathbf{Z}\right) \rightarrow \pi_{s}^{-1}\left(P_{\infty} \mathbf{C}^{+}\right)$. Because $\pi_{s}^{-1}\left(P_{n} \mathbf{C}^{+}\right)$is finite, we can find $\bar{\tau}$ with $\beta(\bar{\tau})=\tau$. Then the $e$-invariant of $\tau$ is given by $h(\bar{\tau})$, where $h: \pi_{s}^{*}(X ; \mathbf{Q} / \mathbf{Z}) \rightarrow K^{*}(X ; \mathbf{Q} / \mathbf{Z})$ is the $K$-theory Hurewicz map, or equivalently by $\bar{\tau}_{*} \in \operatorname{Hom}\left(K_{0}\left(P_{\infty} \mathrm{C}\right), K_{0}(* ; \mathbf{Q} / \mathbf{Z})\right) \simeq K^{0}\left(P_{\infty} \mathrm{C} ; \mathbf{Q} / \mathbf{Z}\right)$ (see [5]). To compute $\bar{\tau}_{*}$ we write $t_{S^{1}}$ as a composition of two transfer maps:

$$
\pi_{n}^{s}\left(P_{\infty} \mathbf{C}^{+}\right) \stackrel{t_{m}}{\rightarrow} \pi_{n+1}^{s}\left(B \mathbf{Z}_{m}^{+}\right) \stackrel{t_{\mathbf{Z}_{m}}}{\rightarrow} \pi_{n+1}^{s}\left(S^{0}\right)
$$

The element $t_{\mathbf{z}_{m}}(1) \in \pi_{s}^{0}\left(B \mathbf{Z}_{m}^{+}\right)$is not in the image of $\beta$, but $t_{\mathbf{Z}_{m}}(1)-m$ is. So $t_{S^{1}}(1)$ and $t_{m}\left(t_{\mathbf{z}_{m}}(1)-m\right)$ differ by $m \cdot t_{m}(1)$.

LEMMA 1.1. For $n$ fixed, there is an $m$ such that $m \cdot t_{m}(1) \in \pi_{s}^{-1}\left(P_{n} \mathrm{C}\right)$ is zero.

Proof. Let $L$ be the universal line bundle over $P_{n}$ C. Then the sphere bundle of $L^{m}$ is the $(2 n+1)$-skeleton of $B Z_{m}$. There exists a number $m$ such that $J\left(L^{m}\right)=0$ in $J\left(P_{n} C\right)$, so $L^{m}$ is orientable for $\pi_{s}^{*}$ and we have an exact Gysin sequence

$$
\rightarrow \pi_{s}^{0}\left(P_{n} \mathrm{C}^{+}\right) \stackrel{\pi^{*}}{\rightarrow} \pi_{s}^{0}\left(\left(B \mathrm{Z}_{m}^{+}\right)^{2 n+1}\right) \stackrel{t_{m}}{\rightarrow} \pi_{s}^{-1}\left(P_{n} \mathrm{C}^{+}\right) \rightarrow
$$

Let pr: $P_{n} \mathrm{C} \rightarrow *$ denote the projection; then $t_{m}^{*}(1)=t_{m}^{\circ} \mathrm{pr}^{*}(1)$ factors over $t_{m} \circ \pi^{*}$, so it must be zero.

Received by the editors June 19, 1978.

AMS (MOS) subject classifications (1970). Primary 55E10, 55B15, 57D90.

Key words and phrases. Transfer, stable homotopy, Lie groups, $e$-invariant, filtration in the Adams spectral sequence. 
So for elements of filtration less than $n$ and $m$ large $\bar{\tau}_{*}$ is given by the composition:

$$
K_{0}\left(P_{\infty} \mathbf{C}\right) \stackrel{t_{m}}{\rightarrow} K_{1}\left(B \mathbf{Z}_{m}\right) \stackrel{\beta}{\leftarrow} \tilde{K}_{2}\left(B \mathbf{Z}_{m} ; \mathbf{Q} / \mathbf{Z}\right) \stackrel{t_{\mathbf{Z}_{m}}}{\rightarrow} K_{2}(* ; \tilde{\mathbf{Q}} / \mathbf{Z})
$$

Proposition 1.2. The element $\left(t_{\mathrm{z}_{m}} \circ \beta^{-1} \circ t_{m}\right) \in \operatorname{Hom}\left(K_{0}\left(P_{\infty} \mathrm{C}\right) ; \mathbf{Q} / \mathrm{Z}\right)$ is given by the Kronecker product with $\operatorname{red}\left(1 /(1-L)-m /\left(1-L^{m}\right)\right) \in K^{-2}\left(P_{\infty} \mathbf{C} ; \mathbf{Q} / \mathbf{Z}\right)$, where red: $K^{*}\left(P_{\infty} \mathrm{C} ; \mathbf{Q}\right) \rightarrow K^{*}\left(P_{\infty} \mathrm{C} ; \mathbf{Q} / \mathbf{Z}\right)$ is the reduction $\bmod \mathbf{Z}$ and $L$ the universal line bundle.

PROOF. We have $t_{\mathrm{z}_{m}} \beta^{-1} t_{m}(z)=\left\langle 1, t_{\mathrm{z}_{m}} \beta^{-1} \circ t_{m}(z)\right\rangle_{K}=$ $\left\langle t_{\mathbf{z}_{m}}(1)-m, \beta^{-1} \circ t_{m}(z)\right\rangle$ where $\langle,\rangle_{K}$ is the Kronecker product $K^{*}(X) \times$ $K_{*}(X ; \mathbf{Q} / \mathbf{Z}) \rightarrow \mathbf{Q} / \mathbf{Z}$. It is well known that $t_{\mathbf{z}_{m}}(1)$ is given by the regular representation of $\mathbf{Z}_{m}$, that is by $t_{\mathbf{Z}_{m}}(1)=\sum_{i=0}^{m-1} \pi^{*}\left(L^{i}\right)$, where $\pi: B Z_{m} \rightarrow P_{\infty} \mathbf{C}$ is the projection. To compute $t_{m} \beta^{-1} \pi^{*}\left(L^{i}-1\right)$, we observe that the transfer $t_{m}$ composed with the Thom isomorphism $\phi$ of the bundle $L^{m}$ is the coboundary map $\delta$ : $K^{1}\left(S\left(L^{m}\right) ; \mathbf{Q} / \mathbf{Z}\right) \rightarrow K^{0}\left(D\left(L^{m}\right), S\left(L^{m}\right) ; \mathbf{Q} / \mathbf{Z}\right)$. This follows from [2], where it is proved that $t_{m}$ is the Umkehr-map of $\pi$ and an easy calculation with Poincaré duality. We therefore consider $\delta \circ \beta^{-1} \circ \pi^{*}$. Let red $^{-1} \circ \delta \circ \beta^{-1}$ be the functional cohomology operation defined by the diagram

$$
\begin{aligned}
& \rightarrow \quad K^{1}(S ; \mathbf{Q}) \quad \rightarrow \quad K^{1}(S ; \mathbf{Q} / \mathbf{Z}) \quad \stackrel{\beta}{\rightarrow} K^{0}(S ; \mathbf{Z}) \quad \stackrel{\text { red }}{\rightarrow} K^{0}(S ; \mathbf{Q}) \\
& \downarrow \delta \quad \downarrow \delta \\
& \rightarrow \quad K^{0}(D, S ; \mathbf{Q}) \stackrel{\text { red }}{\rightarrow} K^{0}(D, S ; \mathbf{Q} / \mathbf{Z}) \quad \rightarrow \quad K^{1}(D, S ; \mathbf{Z}) \quad \rightarrow
\end{aligned}
$$

It is easy to see that this operation is up to a sign the same as the one defined by the diagram

$$
\begin{array}{cccccccc}
\rightarrow & K^{0}(D, S) & \rightarrow & K^{0}(D) & \stackrel{\pi^{*}}{\rightarrow} & K^{0}(S) & \stackrel{\delta}{\rightarrow} & K^{1}(D, S) \\
& \downarrow & & \downarrow q & & \downarrow q & & \\
& K^{0}(D, S ; \mathbf{Q}) & \stackrel{j^{*}}{\rightarrow} & K^{0}(D ; \mathbf{Q}) & \rightarrow & K^{0}(S ; \mathbf{Q}) & \rightarrow
\end{array}
$$

So $\operatorname{red}^{-1} \circ \delta \circ \beta^{-1}(z)=j^{*-1} q \pi^{*-1}(z)$. The element $\left(1-L^{m}\right) /(1-L)=$ $\sum_{i=1}^{m}\left(\begin{array}{c}m \\ i\end{array}\right) \cdot(L-1)^{i-1}$ is invertible in $K^{0}\left(P_{\infty} \mathbf{C} ; \mathbf{Q}\right)$; thus the element $\left(L^{i}-1\right) /(1-$ $\left.L^{m}\right)$ in $K^{0}\left(P_{\infty} \mathbf{C} ; \mathbf{Q}\right)$ is well defined.

Because $j^{*} \circ \phi$ is the cup product with the Euler class $e\left(L^{m}\right)=1-L^{m}$ we have $\phi^{-1} \circ j^{*-1} \circ q\left(L^{i}-1\right)=\operatorname{red}\left(\left(L^{i}-1\right) /\left(1-L^{m}\right)\right)$ and so $t_{m} \beta^{-1} \pi^{*}\left(L^{i}-1\right)=$ $\operatorname{red}\left(\left(L^{i}-1\right) /\left(1-L^{m}\right)\right)$. This gives

$$
\begin{aligned}
t_{m}^{\cdot} \beta^{-1}\left(t_{\mathbf{z}_{m}}(1)-m\right) & =\operatorname{red}\left(\sum_{i=1}^{m-1}\left(L^{i}-1\right) /\left(1-L^{m}\right)\right) \\
& =\operatorname{red}\left(\left(\sum_{i=0}^{m-1} L^{i}-m\right) /\left(1-L^{m}\right)\right) \\
& =\operatorname{red}\left(\left(\left(L^{m}-1\right) /(L-1)-m\right) /\left(1-L^{m}\right)\right) \\
& =\operatorname{red}\left(1 /(1-L)-m /\left(1-L^{m}\right)\right) .
\end{aligned}
$$


THEOREM 1.3. The e-invariant of $\tau$ is given by the power series $1 / x-1 / \log (x+1)$ in $K^{-2}\left(P_{\infty} \mathbf{C} ; \mathbf{Q} / \mathbf{Z}\right)$ where $x=L-1$.

Proof. Given an element $w$ in $\operatorname{im}\left(K_{0}\left(P_{s} C\right) \rightarrow K_{0}\left(P_{\infty} C\right)\right)$ we have

$$
\begin{aligned}
\langle 1 / x-m /(1 & \left.\left.-L^{m}\right), w\right\rangle_{K}=\left\langle\operatorname{ch}(x)^{-1}-m \cdot \operatorname{ch}\left(1-L^{m}\right)^{-1}, \operatorname{ch}(w)\right\rangle_{H} \\
& =\left\langle\left(1-e^{z}\right)^{-1}-z^{-1}, \operatorname{ch}(w)\right\rangle_{H}+\left\langle z^{-1}-m\left(1-e^{m z}\right)^{-1}, \operatorname{ch}(w)\right\rangle_{H}
\end{aligned}
$$

where $z=c_{1}(L)$. The power series of $z^{-1}-m /\left(1-e^{m z}\right)$ shows that for $m$ large the last product becomes integral; so

$$
\left\langle(1-L)^{-1}-m /\left(1-L^{m}\right), w\right\rangle_{K} \equiv\left\langle x^{-1}-1 / \log (x+1), w\right\rangle_{K} \quad \bmod \mathbf{Z} .
$$

The slant product with the element $\omega=x^{-1}-1 / \log (x+1) \in$ $K^{-2}\left(P_{\infty} \mathbf{C} ; \mathbf{Q} / \mathbf{Z}\right)$ defines a map $\hat{t}: K_{0}\left(B T^{n}\right) \rightarrow K_{2}\left(B T^{n-1} ; \mathbf{Q} / \mathbf{Z}\right)$. Because the transfer of the fibre bundle $B T^{n-1} \times E S^{1} \rightarrow B T^{n}$ is induced by the stable map id $\wedge \tau$ we have from Theorem 1.3:

Corollary 1.4. The composition

$$
\pi_{2 m}^{s}\left(B T^{n+1_{+}}\right) \stackrel{t}{\rightarrow} \pi_{2 m+1}^{s}\left(B T^{n_{+}}\right) \stackrel{e_{c}}{\rightarrow} K_{0}\left(B T^{n} ; \mathbf{Q} / \mathbf{Z}\right) / \mathrm{im} H_{2 m}\left(B T^{n} ; \mathbf{Q}\right)
$$

is given by $\hat{t} \circ h$, where $h: \pi_{2 m}^{s}\left(B T^{n+1+}\right) \rightarrow K_{0}\left(B T^{n+1}\right)$ is the Hurewicz map.

For applications of Corollary 1.4 see [5].

Let $p$ be an odd prime. Then some suspension of the $p$-localization of $P_{n} \mathrm{C}$ splits into a wedge of $(p-1)$ spaces

$$
S^{r} \wedge P_{n} \mathbf{C}_{(p)} \simeq X_{1} \vee X_{2} \vee \cdots \vee X_{p-1}
$$

where $X_{i}$ has only cells in dimensions $2 i+2 t(p-1)+r$ (for a proof see [7]). Therefore the stable map $\tau \in \pi_{s}^{-1}\left(P_{\infty} C_{(p)}^{+}\right)$decomposes into a sum of $\tau_{i} \in \pi_{s}^{*}\left(X_{i}\right)$.

Proposition 1.5. Let $p$ be an odd prime, then $e_{c}\left(\tau_{i}\right)=0$ if $i \neq-1(p-1)$.

Proof. The class $x^{-1}-1 / \log (x+1)$ in $K^{-2}\left(P_{\infty} C ; Q\right)$ is mapped under the Chern character into $\left(1-e^{z}\right)^{-1}-z^{-1}=\sum_{i=0}^{\infty}\left(B_{i+1} / i+1\right) \cdot z^{i} / i$ !. So $e\left(\tau_{i}\right)=$ red $\circ \operatorname{ch}^{-1}\left(f_{i}\right)$ where $f_{i}:=\sum_{i=0 ; j i(p-1)}^{\infty}\left(B_{j+1} / j+1\right) z^{j} / j !$ in $H^{*}\left(X_{i} ; \mathbf{Q}\right)$. Now the cannibalistic characteristic class $\rho^{k}: K^{*}(X) \rightarrow K^{*}(X) \otimes \mathrm{Z}[1 / k]$ operates on the $2 n$-sphere as multiplication by $\left(k^{n}-1\right) \cdot B_{n} / n[1]$. It is easy to see that $\psi^{k}-1$ : $K^{*}\left(X_{i} ; \mathbf{Z}_{(p)}\right) \rightarrow K^{*}\left(X_{i} ; \mathbf{Z}_{(p)}\right)$ is an isomorphism for $k \neq 0(p)$ and $i \neq-1(p-1)$. So $\rho^{k} \circ\left(\psi^{k}-1\right)^{-1}(x)$ is a well-defined class in $K^{-1}\left(X_{i} ; \mathbf{Z}_{(p)}\right)$. If $x$ is the class of $L-1$ in $K^{*}\left(X_{i} ; \mathbf{Z}_{(p)}\right)$, then $\operatorname{ch} \rho^{k} \circ\left(\psi^{k}-1\right)^{-1}(x)=f_{i}$ so $e\left(\tau_{i}\right) \equiv 0 \bmod \mathbf{Z}_{(p)}$.

A basis of $H^{2}\left(B T^{n} ; Z\right)$ defines a homeomorphism $g: B T^{n} \rightarrow P_{\infty} \mathbf{C}$ $\times \cdots \times P_{\infty} \mathrm{C}$. Using $g$ we decompose the suspension of $B T^{n}$ into a wedge of smash products of $P_{\infty} \mathrm{C}$ and so, after localization, into a wedge of smash products of the $X_{i}$.

By (2.1) of [5] and Proposition 1.5 we find that the transfer $t: \pi_{*}^{s}\left(B T^{n_{+}}\right)_{(p)} \rightarrow$ $\pi_{*}^{s}\left(S^{0}\right)_{(p)}$ is concentrated on the component $\pi_{*}^{s}\left(X_{r} \wedge X_{r} \wedge \cdots \wedge X_{r}\right)$ (n factors with $r \equiv-1(p-1))$. That is to say, only on this component can $t$ raise the filtration associated to the BP-Adams spectral sequence by $n$. On all other components $t$ must raise the filtration at least by $n+2(p-1)$. 
Given an element $(B, f) \in \pi_{2 m}^{s}\left(B T^{n}\right)_{(p)}$ we can use the Hurewicz map $h: \pi_{2 m}^{s}\left(B T^{n}\right) \rightarrow H_{2 m}\left(B T^{n}\right)$ to find out when $(B, f)$ has a component in $\pi_{*}^{s}\left(X_{r} \wedge X_{r} \wedge X_{r}\right)$ up to higher filtration. Because the filtration increases in steps of $2(p-1)$ on spaces like $X_{r} \wedge X_{r} \wedge \cdots \wedge X_{r}$ we have:

Corollary 1.6. Given $z \in \pi_{2 m}^{s}\left(B T^{n}\right)_{(p)}$ with $h(z)=0$ in $H_{*}\left(X_{r} \wedge \cdots \wedge X_{r}\right)$, then $t(z) \in \pi_{*}^{s}\left(S^{0}\right)_{(p)}$ is at least of filtration $n+2(p-1)$.

2. Application to Lie groups. Let $G$ be a compact Lie group of rank $n$ with maximal torus $T$. The left invariant framing $\mathcal{L}$ of $G$ induces a framing $\mathcal{L}^{*}$ of $G / T$. Together with the classifying map $f$ of the bundle $G \rightarrow G / T$ we get an element $\left[G / T, f, \mathcal{L}^{*}\right] \in \Omega_{*}^{\mathrm{fr}}\left(B T^{n}\right) \cong \pi_{*}^{s}\left(B T^{n_{+}}\right)$. The image of this element under the transfer $t: \pi_{*}^{s}\left(B T^{n_{+}}\right) \rightarrow \pi_{*}^{s}\left(S^{0}\right)$ is the element $[G, \mathcal{E}]$ defined by the Lie group in $\pi_{*}^{s}\left(S^{0}\right)$ [5].

Let $z_{1}, \ldots, z_{n}$ be a basis of $H^{2}\left(B T^{n} ; \mathbf{Z}\right)$. Then the image of $\left[G / T, f, \mathcal{L}^{*}\right]$ under the Hurewicz map $h: \pi_{*}^{s}\left(B T^{n_{+}}\right) \rightarrow H_{*}\left(B T^{n}\right)$ is determined by the Kronecker products

$$
c_{(k)}=\left\langle f^{*}\left(z_{1}^{k_{1}} \cup \cdots \cup z_{n}^{k_{n}}\right),[G / T]\right\rangle_{H}
$$

where $2 \Sigma_{i} k_{i}=\operatorname{dim} G / T$. Let $x_{1}, \ldots, x_{n}$ be a basis of $H^{1}\left(T^{n} ; \mathbf{Z}\right)$ and $\tau$ : $H^{1}\left(T^{n} ; \mathbf{Z}\right) \rightarrow H^{2}(G / T ; \mathbf{Z})$ the transgression map. We can choose $z_{1}, \ldots, z_{n}$ such that $\tau\left(x_{i}\right)=f^{*}\left(z_{i}\right)$. In the following we will identify $H^{1}\left(T^{n} ; Z\right)$ with the dual of the integer lattice of $G$.

A set of $n$ linearly independent elements in $H^{2}(G / T ; Z)$ defines a lattice $\Gamma$ in $H^{2}(G / T)$ and a torus bundle $E \rightarrow G / T$. The total space $E$ is then the quotient of $G$ by a finite group $H$ with order $|H|=$ index of $\Gamma$. (The manifold $E$ is framed in a canonical way.) In considering the $p$-component only, we really do not need a basis of $H^{2}\left(B T^{n} ; \mathbf{Z}\right)$ but only one of $H^{2}\left(B T^{n} ; \mathbf{Z}_{(p)}\right)$ because the use of $m \cdot z_{i}$ for $m \in \mathbf{Z}$ means that we turn from $G$ to the framed manifold $\bar{G}=G / \mathbf{Z}_{m}$. But if $m \neq 0(p)$ then $m \cdot[\bar{G}, \overline{\mathcal{E}}]_{(p)}=[G, \mathcal{E}]_{(p)}$.

Proposition 2.1. Let $p>3$ be a prime and $G$ a compact Lie group of rank $n$. Then there exists a decomposition of $S B T_{(p)}^{n}$ such that the component of $h\left(\left[G / T, f, \mathcal{L}^{*}\right]\right)$ in $H_{*}\left(X_{r} \wedge X_{r} \wedge \cdots \wedge X_{r}\right) \quad(r \equiv-1(p-1), n$ factors $)$ is zero.

Proof. We only need to prove that for all primes $p>3$ and all compact Lie groups $G$ there exists a basis $z_{1}, \ldots, z_{n}$ such that the numbers $c_{(k)}$ with all $k_{i} \equiv-1(p-1)$ vanish. The general argument is as follows: First let $G$ be simple. We look for classifying elements $f^{*}\left(z_{i}\right)=\gamma_{i}$ such that there exist for each pair $(i, j)$, $i \neq j$, an element $w$ in the Weyl group of $G$ which permutes $\gamma_{i}$ and $\gamma_{j}$, leaves all the others fixed and operates on the fundamental class $[G / T]$ as multiplication by -1 . Let $z=\gamma_{1}^{k_{1}} \cup \cdots \cup \gamma_{n}^{k_{n}}$ with $k_{i} \equiv-1(p-1)$. If $\langle z,[G / T]\rangle \neq 0$ then all $k_{i}$ must be different, for if $k_{i}=k_{j}$ with $i \neq j$, we have a $w \in W(G)$ with $w^{*}\left(\gamma_{i}\right)=\gamma_{j}$, that is $w^{*}(z)=z$; but $w_{*}[G / T]=-[G / T]$. By calculating the dimension of such $z$ we then see that all the corresponding $c_{(k)}$ must vanish. For a semisimple Lie 
group we look for such elements in each simple component. We call such a set of classifying elements a *-basis.

The existence of $a^{*}$-basis can be easily checked for the simply connected simple Lie groups:

1. $A_{n}, B_{n}, C_{n}, D_{n}$. We can use as a *-basis the elements denoted by $\varepsilon_{i}$ in [4]. For the dimension argument to hold for $B_{n}$ and $C_{n}$ we must suppose $p>3$ whereas $p>2$ suffices for $A_{n}$ and $D_{n}$.

2. For $G_{2}$ see [3].

3. For $F_{4}$ we refer to [3]. It is an exercise to see that with respect to the given basis in [3] there are only the possibilities $(1,5,7,11),(1,3,9,11),(3,5,7,9)$, $(1,3,7,13)$, and $(1,3,5,15)$ for exponent sequences $k_{i}$.

4. The cases $E_{6}, E_{7}, E_{8}$ can be treated using exercises 29 and 30 in Chapter 4 of [9].

Now let $G$ be semisimple. Then $G$ is the quotient of a product of simple Lie groups by a finite subgroup of the center of this product. Because we have $p>3$ we only have to consider subgroups of the center of a product of $\operatorname{SU}\left(n_{i}\right)$ 's.

Let $\tilde{G}=\operatorname{SU}\left(n_{1}\right) \times \cdots \times \operatorname{SU}\left(n_{m}\right), H \subset \operatorname{center}(\tilde{G}), G=\tilde{G} / H$ and set $\bar{G}=$ $\tilde{G} / \operatorname{center}(\tilde{G})$. We denote the dual of the integer lattice of a Lie group $G$ by $I_{G}^{*}$. To the covering $\tilde{G} \rightarrow G$ there corresponds an inclusion of lattices $I_{G}^{*} \subset I_{G}^{*}$. Contained in $I_{G}^{*}$ is $I_{G}^{*}$. It suffices to find a ${ }^{*}$-basis for $I^{*} \otimes \mathrm{Z}_{(p)}$ for all lattices $I^{*}$ between $I_{G}^{*}$ and $I_{\tilde{G}}^{*}$. The lattices $I_{\tilde{G}}^{*}$ and $I_{\bar{G}}^{*}$ are product lattices. Using the notation of [4] we have $I_{G}^{*}=\prod_{i=1}^{m}\left\langle\varepsilon_{1}^{i}, \ldots, \varepsilon_{n_{i}-1}^{i}\right\rangle$ and $I_{G}^{*}=\prod_{i=1}^{m}\left\langle\varepsilon_{1}^{i}-\varepsilon_{2}^{i}, \ldots, \varepsilon_{1}^{i}-\varepsilon_{n_{i}}^{i}\right\rangle$ (we set $\Sigma_{k} \varepsilon_{k}^{i}$ $=0$ ).

Let $F=I^{*} / I_{G}^{*}$ and $\pi: I^{*} \rightarrow F$ be the projection. We can suppose that $F$ is a $p$-group. Let $z_{1}, \ldots, z_{f}$ be preimages of the generators of the factors of $F$ under $\pi$. Because of $\pi\left(\varepsilon_{j}^{i}\right)=\pi\left(\varepsilon_{k}^{i}\right)$ we can write $z_{j}$ as a linear combination of the $\varepsilon_{1}^{i}$ : $z_{j}=\sum_{i=1}^{m} a_{i}^{j} \cdot \varepsilon_{1}^{i}$. We have $n_{j} \cdot \varepsilon_{1}^{j} \in I_{\bar{G}}^{*}$. Furthermore we can choose the $z_{j}$ in such a way that $a_{i}^{j}=0$ for $i<j$ and $\nu_{p}\left(n_{k} / a_{k}^{k}\right)>\nu_{p}\left(n_{j} / a_{j}^{k}\right)$ for $j>k$ and $k=1, \ldots, f$. It is then clear that $I^{*}=I_{G}^{*}+\left\langle z_{1}, \ldots, z_{f}\right\rangle$.

We define the lattice $\Gamma$ to be generated by $\varepsilon_{1}^{1}+z_{1}-\varepsilon_{2}^{1}, \ldots, \varepsilon_{1}^{1}+z_{1}-\varepsilon_{n_{1}}^{1}$, $\varepsilon_{1}^{2}+z_{2}-\varepsilon_{2}^{2}, \ldots, \varepsilon_{1}^{f}+z_{f}-\varepsilon_{n_{j}}^{f}, \ldots, \varepsilon_{1}^{m}-\varepsilon_{n_{m}}^{m}$. These elements form a *-basis for $\Gamma$. It is easy to see that $\Gamma \otimes \mathbf{Z}_{(p)}=I^{*} \otimes \mathbf{Z}_{(p)}$.

In the general case, where $G$ is not semisimple, it is clear that there is always at least one basis element of $H^{1}\left(T^{n} ; Z\right)$ lying in the kernel of the transgression map. So there is no nonzero $c_{(k)}$ in which all classifying elements appear.

COROLlaRY 2.2. Let $p>3$ be a prime and $G$ a compact Lie group of rank $n$. Then the p-component of $[G, \mathcal{E}]$ in $\pi_{*}^{s}\left(S^{0}\right)$ is at least of filtration $n+2(p-1)$ where the filtration is associated to the Adams spectral sequence for $B P$.

The vanishing line of the $E^{2}$-term of the Adams spectral sequence for BP (see [6]) shows that elements of high filtration cannot exist in low dimensions. This and (2.2) and some simple dimension arguments show that in the known part of stable homotopy-see for example [8]-we have $[G, \mathcal{L}\}_{(p)}=0$ for $p>3$. This also shows that $\left[G, \mathcal{L} l_{(p)}\right.$ for an exceptional Lie group $G$ can be nonzero only for $p=2$ or 3 . 


\section{REFERENCES}

1. J. F. Adams, On the groups J(X). II, Topology 3 (1965), 137-171.

2. J. M. Boardman, Stable homotopy theory, duality and Thom spectra (mimeographed notes), Chapter 5, University of Warwick, 1966.

3. A. Borel and F. Hirzebruch, Characteristic classes and homogeneous spaces. II, Amer. J. Math. 81 (1959), 315-382.

4. N. Bourbaki, Groupes et algèbres de Lie, Hermann, Paris, 1968.

5. K. Knapp, Rank and Adams filtration of a Lie group, Topology 17 (1978), 41-52.

6. H. R. Miller, Some algebraic aspects of the Adams-Novikov spectral sequence, Dissertation, Princeton Univ., Princeton, N. J., 1974.

7. M. Mimura, G. Nishida and H. Toda, Mod p decomposition of compact Lie groups, Publ. Res. Inst. Math. Sci. Kyoto Univ. 13 (1977), 627-680.

8. S. Oka, The stable homotopy groups of spheres. III, Hiroshima Math. J. 5 (1975), 407-438.

9. V. S. Varadarajan, Lie groups, Lie algebras and their representations, Prentice-Hall, Englewood Cliffs, N.J., 1974.

MAthematisches Institut der Universitüt BonN, Wegelerstrasse 10, D-5300 BonN, Federal REPUBlic OF Germany 